\title{
Equilibrium Molecular Interactions in Pure Gases
}

\author{
Boris I. Sedunov \\ The Information Systems and Computer Technologies Department, Russian New University, Radio St. 22, Moscow 105005, Russia
}

Correspondence should be addressed to Boris I. Sedunov, tropic1@ya.ru

Received 3 September 2011; Revised 13 November 2011; Accepted 21 November 2011

Academic Editor: Juan Ortega

Copyright () 2012 Boris I. Sedunov. This is an open access article distributed under the Creative Commons Attribution License, which permits unrestricted use, distribution, and reproduction in any medium, provided the original work is properly cited.

\begin{abstract}
The equilibrium molecular interactions in pure real gases are investigated based on the chemical thermodynamics principles. The parallels between clusters in real gases and chemical compounds in equilibrium media have been used to improve understanding of the real gas structure. A new approach to the equilibrium constants for the cluster fractions and new methods to compute them and their significant parameters from the experimental thermophysical data are developed. These methods have been applied to some real gases, such as Argon and Water vapors and gaseous Alkanes. It is shown that the four-particle clusters make a noticeable contribution in the thermophysical properties of the equilibrium Water vapor. It is shown also that the effective bond energy for dimers in Alkanes linearly grows with the number of carbon atoms in the molecule.
\end{abstract}

\section{Introduction}

Modern technologies benefit from the utilization of extra pure and highly uniform materials and substances and precise knowledge of their thermophysical properties. For computer-aided design and control of technological processes, precise models of thermophysical properties and equations of state for pure gases are very important because the real gases are widely used both as initial and intermediate materials in modern technologies. The models for pure gases can be used to build more complex models for mixtures of different gases. This work has a goal to show no obvious relations between equilibrium molecular interaction parameters in pure real gases and to find useful expressions to predict equilibrium properties of pure gases in changing external conditions. The work is based on a phenomenological approach that, nevertheless, provides a vision of the real gas structure on a microscopic level.

The paper tries to answer the following questions, arising in courses of molecular and chemical physics.

(i) What are the equilibrium constants and bond energies for real gas clusters considered as the low bond energy analogues of chemical compounds?

(ii) How to define the bond energies for clusters that in contrast to a wide separation of quantum levels of compounds have a dense spacing of multiple energetic levels?

(iii) How to build the bridge between the virial equations of state based on variables that are sums of partial quantities for cluster fractions and the chemical equilibrium law, where arguments for concentrations of chemical compounds are the partial concentrations of reacting components [1]?

(iv) How to transform the Sackur-Tetrode equation for entropy $[2,3]$ of ideal atomic gases for real gases with account of the molecular interactions and internal movements?

The paper intends to show that clusters in equilibrium real gases obey to laws that differ from the well-known laws for chemical compounds equilibrium [1]. One of ways to understand the properties of real gases is their comparison with chemical media, where dimerized compounds, such as Alkali metal $[4,5]$ or Nitrogen Dioxide $[1,6]$ dimers, may be formed in equilibrium conditions from their monomers. While the concentrations of chemical compounds and their equilibrium constants may be found from spectroscopic measurements, the thermal movement shadows and erodes the spectral lines of clusters. There are many papers on spectroscopic investigation of molecular clusters in molecular beams, but not in equilibrium gases. There are many works 
on molecular dynamics simulation of gaseous clusters, which do not build the bridge between the molecular interaction parameters and the thermophysical properties of real gases. This work develops the reverse methods to estimate the bond parameters of clusters in pure real gases from thermophysical data.

There is a productive way to the clusters' equilibrium constants and molecular interaction parameters, based on the processing of known thermophysical data. But found so the cluster fractions' apparent equilibrium constants responsible for the Pressure-Density-Temperature relations (PDT equilibrium constants) are essentially different from the potential-energy (PE-) based equilibrium constants in real gases, while this difference for chemical compounds may be negligible. The paper strives to explain these facts.

This paper deals with the extension of the thermodynamics to real gaseous systems. All conclusions of the developed here theory are verified with the utilization of precise experimental data from the NIST (the National Institute of Standards and Technology USA) databases [7, 8]. Now, to provide the computer-aided design and quality control for many technological processes, comprehensive databases of thermophysical properties for every used substance are accumulated and regularly updated. In modern science and technology, their creation goes on constantly with a steady growth of the data abundance and precision. Constant improvement of comprehensive and precise databases of the NIST $[7,8]$ is the best example of this kind of scientific activity. The systematization of experimental data, from one side, provides practical application, testing, and correction of the existing theoretical models and methods but, from the other side, opens possibilities for further development of the theoretical basis. This paper shows how these possibilities work.

\section{The Equilibrium Structure of Pure Gases}

2.1. Clusters and Monomers in Real Gases. Real gas consists of basic particles (atoms or molecules), interacting through their interaction potential [1,9-11]. The main part of gas consists of monomers that are the free moving basic particles performing elastic interactions with other particles without falling in bound states. Due to the attraction part of the interaction potential, some basic particles collect in temporary stable agglomerations with different numbers of particles, Figure 1. These agglomerations are named clusters [12-15] that move in a gas-like free particles and sometimes collide with other clusters or monomers. In spite of the plurality of clusters existing in a real gas, all equilibrium thermophysical properties of real gases are estimated for a fixed number of basic particles, in this paper, for a mole. Due to constantly changing interactions between basic particles in a real gas, it is difficult to define correctly the monomers and clusters from the microscopic point of view.

In this paper, the thermophysical properties of the monomer and cluster fractions are found by processing the known thermophysical properties for all real gas. The results of this processing are not obvious; they reflect both elastic and no elastic interactions between basic particles.

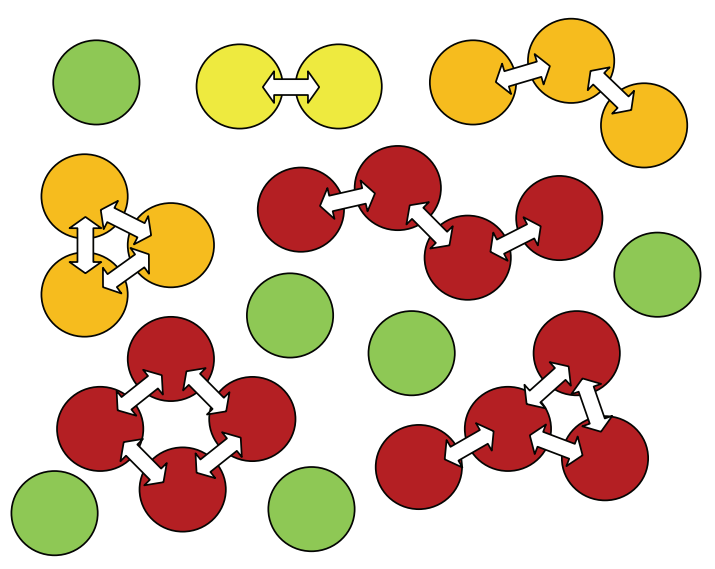

FIGURE 1: Schematic view of the real gas structure: the basic particles of the real gas (balls of all colors) in forms of monomers (green balls) and clusters (other colors). Yellow balls: dimer; orange balls: trimers with open chain and closed isomer configurations; red balls: tetramers (quatromers) with open chain, closed, and partly closed structures; white arrows: intermolecular bonds.

Figure 1 presents the schematic view of the real gas structure that is used here only for interpretation of the computeraided analysis of experimental data, but not for microscopic computations of the real gas properties.

Figure 1 does not show all types of the 4-particle isomers and does not show larger clusters at all. Not to overload the picture, there is not shown the tetragonal configuration of the tetramer with 6 bonds between particles. But the figure clearly shows that different isomers at the same number of particles in a cluster can possess different numbers of bonds. The number of bonds characterizes the cluster's bond energy, and the isomer configuration determines the cluster's entropic factor, connected with the number of states in this configuration. Two of these factors are responsible for prevailing of some types of isomer structures at a given temperature and for a change of the prevailing types with a change of temperature. It will be shown how interaction parameters found by phenomenological analysis of the experimental data point out on the prevailing types of isomers.

The collisions in a gas can be divided on elastic and no elastic types. After an elastic collision, the numbers of basic particles in colliding clusters and their isomer configurations stay not changed. Figure 1 shows only the bonds between basic particles resulting from the no elastic collisions but does not show the elastic forces between particles that influence significantly the thermophysical properties of real gases. Clusters at any type of collisions, even at the no elastic, conserve their common momentum. Two colliding monomers perform only the elastic collision and after this collision fly apart again as monomers. To form the bound state of a dimer, the two monomers at their meeting should collide also with the third particle (monomer or cluster) to give off the excess of their common kinetic energy.

Generally speaking, the clusters are the products of the no elastic collisions, in which the cluster's bound state is 
formed by transferring the excess kinetic energy to some third particle. After the no elastic collision, the colliding particles fall into potential wells created by the attraction part of molecular interactions, Figure 2.

Figure 2 shows principal differences between molecular interactions in clusters and chemical compounds.

(i) The potential well for particles in a cluster is not as deep as in a chemical compound that shortens the clusters' lifetimes by many orders of magnitude as compared to chemical compounds.

(ii) The average width of the potential well in a cluster is much larger than in a chemical compound that lowers distances between energetic levels in clusters by many orders of magnitude as compared to chemical compounds.

(iii) The average numbers of bound states in clusters are many orders of magnitude larger than the numbers of bound states in chemical compounds.

(iv) The potential well around one gaseous particle may attract several particles, but the chemical potential well may disappear for the third particle after formation of the two-particle compound.

Particles in clusters and compounds do not occupy the position at the minimum of their potential. In gases, the thermal movement lifts the particles to higher levels, but, in compounds, their ground level stays over the potential minimum due to quantum laws. We should develop the method of finding the average energy of clusters in their bound states as an equivalent to the Schrodinger equation for the ground level of chemical compounds.

2.2. The Equilibrium Structure of Real Gases. The real gas consists of cluster fractions with a fixed number $n$ of basic particles and with a definite isomer structure of bonds. The equilibrium structure of a real gas can be expressed through the cluster fractions' populations. The cluster fractions keep dynamic equilibrium of their populations through basic particles exchange. Unlike crystals, where structure means the configuration of the elementary cell, regularly repeated in space [16], the equilibrium structure of gases is disordered but statistically stable and determining properties of real gases. Due to differences between the isomers' energy and entropy factors, some isomer configurations are prevailing at a given temperature. The change of prevailing types of isomers is responsible for the temperature dependences of the clusters' molecular interaction parameters that may be found from the equilibrium thermophysical properties of gases.

The bonds between basic particles in real gases are much weaker than the chemical bonds, how it is reflected by the Figure 2. Their values in most cases are comparable with the energy of thermal movement; therefore, the relaxation time to establish the cluster fractions populations is much lower than the typical times for experimental determination of thermophysical properties of gases. But this rule may be not valid for overcooled gases and for the sound speed or the complex dielectric permeability measurements.
In this work, the main variable for the computer-aided analysis of thermophysical properties is the molar density of the monomer fraction [17-19], $D_{m}$. The values of the monomer fraction density can be found from the isothermal pressure dependence of the total density $D$.

\subsection{Monomer Fraction Density and the Chemical Potential.} Due to a fast time to establish equilibrium in the cluster fractions populations, the Gibbs energy for a mole of basic particles (the chemical potential), G, does not depend on their aggregate state: the molar Gibbs energy of basic particles in each cluster fraction, $G_{n}$, and in the monomer fraction, $G_{m}$, is the same and is equal to the total Gibbs energy of the gas: $G_{n}=G_{m}=G[17,18]$. This statement is correct only for equilibrium systems. In a no equilibrium situation, the basic particles' Gibbs energies for different cluster fractions may split, like the Fermi quasilevels for electrons and holes in semiconductors.

For atomic ideal gases, the molar Gibbs energy $G=$ $-R T \ln \left(V / V_{q}\right)$, where $V$ is the molar volume of gas and $V_{q}$ is the molar quantum volume of atoms [20]. In molecular gases, we should add the part of the molar Gibbs energy responsible for the internal molecular movements: rotations of molecules and vibrations of their atoms, $G_{0}$ [18].

The monomer fraction in a gas behaves as an ideal gas with a molar volume $V_{m}=1 / D_{m}$; therefore, the molar Gibbs energy $G$ of basic particles is [18]:

$$
G=G_{m}=G_{0}+R T \ln \left(D_{m} V_{q}\right),
$$

where $V_{q}$ is the molar quantum volume for basic particles that is proportional to the third degree of the thermal de Broglie wavelength:

$$
V_{q}=\frac{h^{3} N_{A}^{4}}{(2 \pi M R T)^{3 / 2}},
$$

where $h$ is the Plank's constant, $M$ is molecular weight of basic particles in $\mathrm{kg} / \mathrm{mol}, N_{A}$ is Avogadro number, and $R$ is universal gas constant. The compact form of (1) combines in $V_{q}$ [20] the entropy dependence on atomic weight, temperature, and Plank constant.

Equation (1) is the thermodynamics-based definition of the monomer fraction density. This equation reminds the well-known equation for fugacity $f: G=B(T)+R T \ln (f)$, suggested by Lewis [21] to solve the problems of no ideality of real gases. The monomer fraction density is more evident: it has a clear statistical and mechanical sense. Its introduction may help in a better understanding of properties of widely used variables: fugacity and activity.

In this theory, $G_{0}$, like $B(T)$ in the theory of fugacity [21], is supposed to be independent on the density and aggregate state of basic particles. All possible changes in the internal movements' part of the Gibbs energy are accounted for by the pressure dependence of $D_{m}$. 10]:

$G$ corresponds to the thermodynamics equation $[1,9$,

$$
\left.\left(\frac{\partial G}{\partial P}\right)\right|_{T}=V
$$



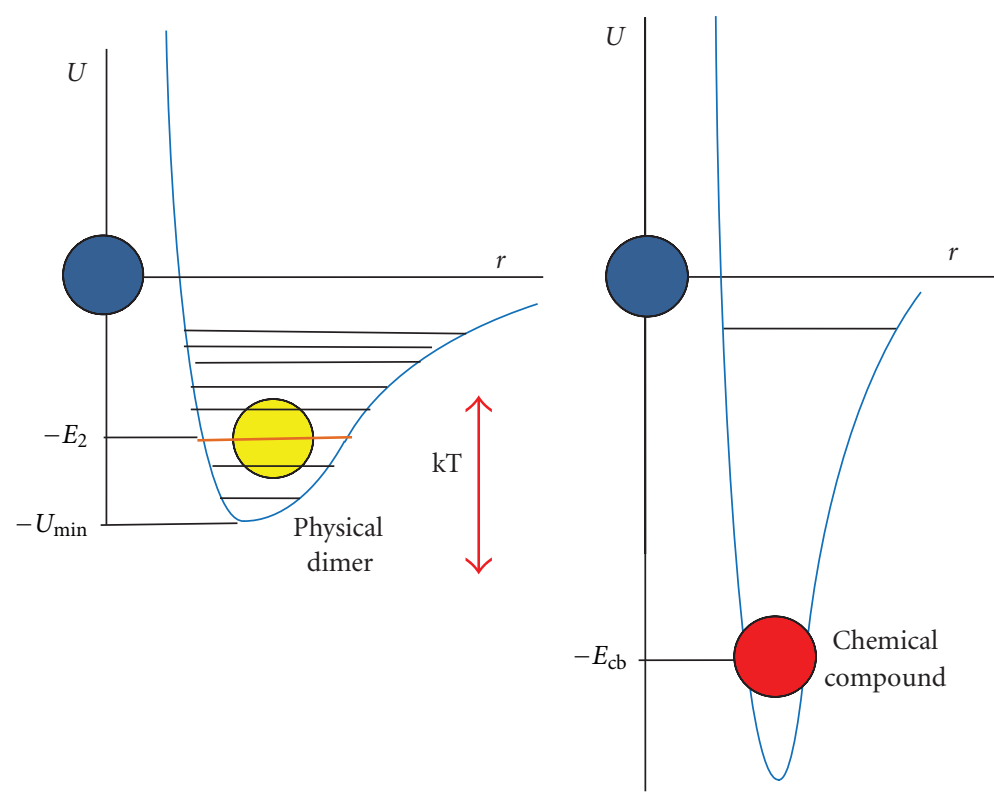

FIGURE 2: Comparison of a cluster with a chemical compound. Blue lines: molecular interaction potentials $U(r)$; narrow lines: energetic levels densely spaced in the cluster and widely separated in the chemical compound. $E_{2}$ : the cluster's effective bond energy, comparable with $k T ; E_{\mathrm{cb}}$ : the chemical bond energy, greatly exceeding the $k T$ value.

that together with (1) leads to the differential equation [17, 18] for $D_{m}$ :

$$
\left.\left(\frac{\partial D_{m}}{\partial P}\right)\right|_{T}=\frac{D_{m}}{R T D}
$$

The numeric integration of (4) should start from such a small pressure $P_{1}$, at which the difference between the real density $D_{1}$ and the ideal density $D_{p 1}=P_{1} / R T$ is small. Then, the initial condition for numeric integration of (1) may be $D_{m 1}=2 D_{p 1}-D_{1}$. Equation (4) is valid for all fluid phases: gaseous, liquid, and supercritical. Figure 3 shows the $D_{m}$ pressure dependence in comparison with the total density dependence at subcritical and supercritical temperatures in Nitrogen.

The figure shows that the share of monomers in the total density falls with growing pressure, but still remains large both in liquid and supercritical media. The integration over the gas-liquid phase transition boundary does not put any problem, because the monomer fraction density does not change its value at the phase transition. When the monomer fraction density is found, it becomes possible to use it as an argument for other thermodynamics functions, such as the entropy or potential energy, and to use it as an argument in series expansions of different values for properties of real gases.

\subsection{Generalization of the Sackur-Tetrode Equation for Real} Gases. The theory of Sackur [2] and Tetrode [3] for entropy of ideal atomic gases had been created in 1912 at the very beginning of the quantum theory development and when the discussion around the third law of thermodynamics still had not been finished. In spite of its applicability only to ideal atomic gases, it made a significant contribution both in thermal and quantum physics. Belloni wrote [22] that Fermi came to the famous Fermi-Dirac statistics trying to find his own way to the Sackur-Tetrode equation. The theory was confirmed experimentally by calculation of the entropy for dilute Neon gas taking into account the third law of thermodynamics. The measurements started from the near zero Kelvin temperature and included solid and liquid phases.

According to this theory, entropy of an ideal atomic gas with a molar volume $V$ equals to

$$
S=2.5 R+R \ln \left(\frac{V}{V_{q}}\right),
$$

where $V_{q}$ is the molar quantum volume given by (2).

Equation (5) with account of (1) and $G=H-T S$ can be transformed to

$$
G-G_{0}=R T \ln \left(D_{m} V_{q}\right)=H-H_{0}-T\left(S-S_{0}\right),
$$

where $H_{0}$ and $S_{0}$ are the enthalpy and entropy of the internal molecular movements: rotations and vibrations. Thus, we come to the Generalized Sackur-Tetrode equation [17-19]:

$$
S=S_{0}+\frac{H-H_{0}}{T}-R \ln \left(D_{m} V_{q}\right)
$$

To test the developed here theory, we compared the experimental values for entropy of Water in gaseous and liquid phases with computed by (7) model values; $D_{m}$ was found by integration of (4). All experimental data have been taken from the NIST database [7].

Figure 4 shows the pressure dependence of the Water entropy in gaseous and liquid phases at a temperature $350 \mathrm{~K}$ and the multiplied by factor $10^{9}$ deviation of the model 


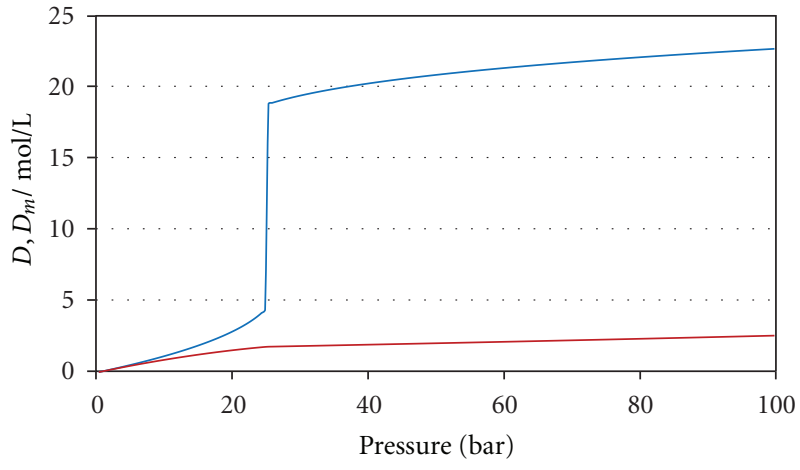

(a)

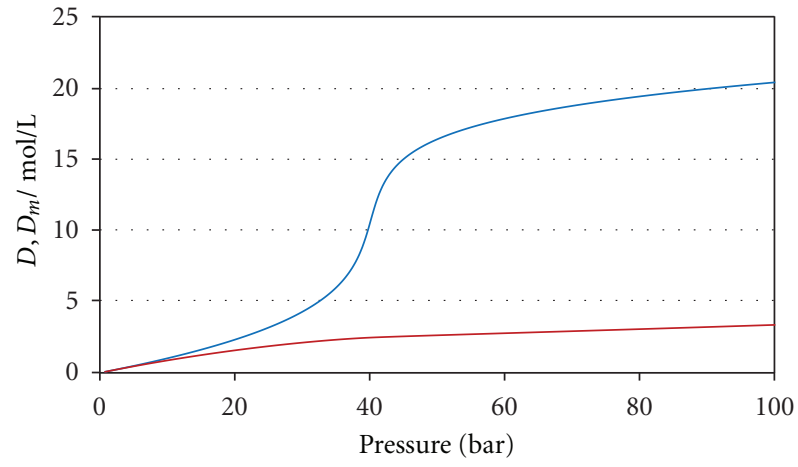

(b)

FIGURE 3: Density (blue lines) and monomer fraction density (red lines) versus pressure in gaseous, liquid, and supercritical Nitrogen at (a) subcritical temperature, $T=120 \mathrm{~K}$; (b) supercritical temperature, $T=130 \mathrm{~K}$.

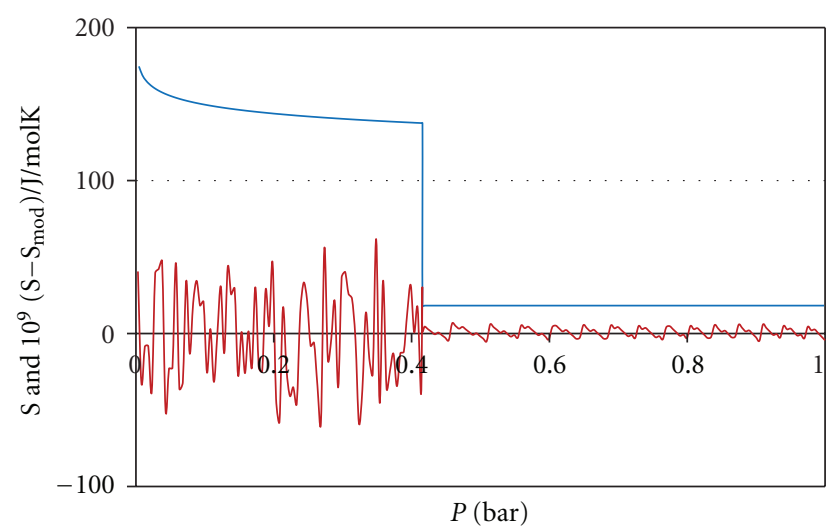

Figure 4: Confirmation of a high precision of the entropy computation by the generalized Sackur-Tetrode equation for Water in the gaseous and liquid phases at temperature $350 \mathrm{~K}$. Blue line: experimental and computed values for entropy; red line: multiplied by factor $10^{9}$ deviations of the computed values from the experimental ones.

values from the experimental data. For $H_{0}$, the result of extrapolation to zero pressure of the experimental values for $H\left(P_{i}\right)$ was taken. For $S_{0}-R \ln \left(V_{q}\right)$ value, the averaged difference between experimental values for $S$ and computed values for $\left(H-H_{0}\right) / T-R \ln \left(D_{m}\right)$ was taken. So this test estimates only the spread of computations. Nevertheless, the level of spread is nine orders of magnitude lower than the total entropy change that tells about correctness of the theory and about the high precision of computations. This high precision of computations results from remarkable features of the NIST database [7]:

(i) the experimental data have been interpolated with a very high precision;

(ii) the data for different thermophysical properties have been mutually correlated.

This test had been applied to a number of pure gases in different conditions, including gaseous, liquid, and supercritical phases, and the results confirm the high precision of computations of the $D_{m}(P, T)$ and $S(P, T)$ values. On this basis, the $D_{m}$ values in a couple with the NIST tables [7] permit to find the cluster fractions equilibrium constants and molecular interaction parameters for pure real gases with a good precision.

\section{Monomer Fraction Density as a New Variable for Thermophysical Functions}

3.1. The Partial Potential Energies for Cluster Fractions in Gases. The potential energy $U(T, P)$ of a real gas can be defined as the difference between the molar internal energy $E(T, P)$ of a real gas and its limit $E_{0}(T)$ at zero pressure:

$$
U(T, P)=E(T, P)-E_{0}(T) .
$$

The $E_{0}(T)$ corresponds to the internal energy for a mole of an ideal gas. In terms of clusters, the potential energy of real gases is equal to a sum of partial potential energies of the cluster fractions with account of the partial kinetic energies change with a change of density.

The contribution $\Delta U_{\mathrm{uv}}$ of chemical compounds, such as the alkali metal $[4,5]$ or the Nitrogen Dioxide $[1,6]$ dimers, with their chemical bond energies $E_{\mathrm{cb}}$, molar densities $D_{d}$, and equilibrium constants $C_{d}$, to the total equilibrium potential energy of a unit volume is equal to

$$
\Delta U_{\mathrm{uv}}=-E_{\mathrm{cb}} D_{d}=-E_{\mathrm{cb}} C_{d} D_{m}^{2} .
$$

The molar densities of dimers $D_{d}$ and monomers $D_{m}$ for chemical compounds can be found from the spectroscopy data. And, on this basis, it is possible to find the equilibrium constants $C_{d}[4,6]$. But partial densities for clusters in real gases cannot be found in this way, and we have to use the potential energy to derive them. Instead of the molar potential energy $U$, we expand the potential energy $U D$ of a unit volume by the monomer fraction density. This expansion does not separate the contributions of different isomers with the same number $n$ of particles in the cluster fraction. In the result, we find the clusters' PE equilibrium constants as the thermodynamically averaged values for all possible isomers at a constant number $n$. 


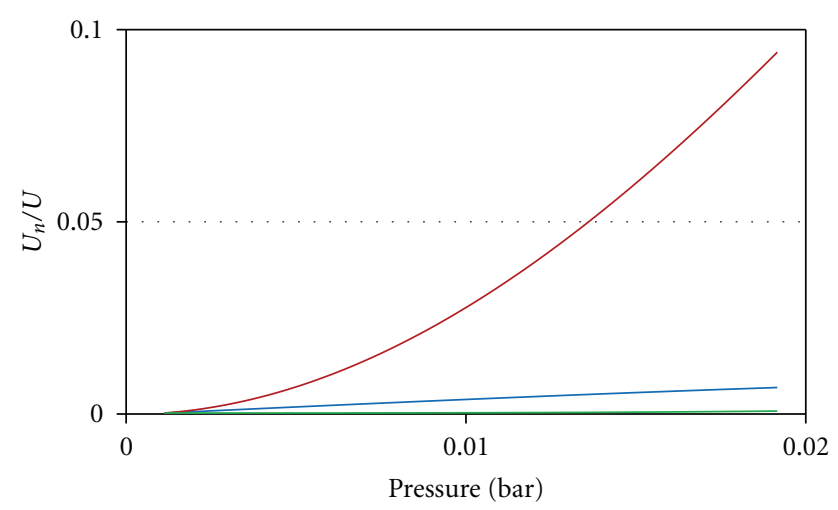

(a)

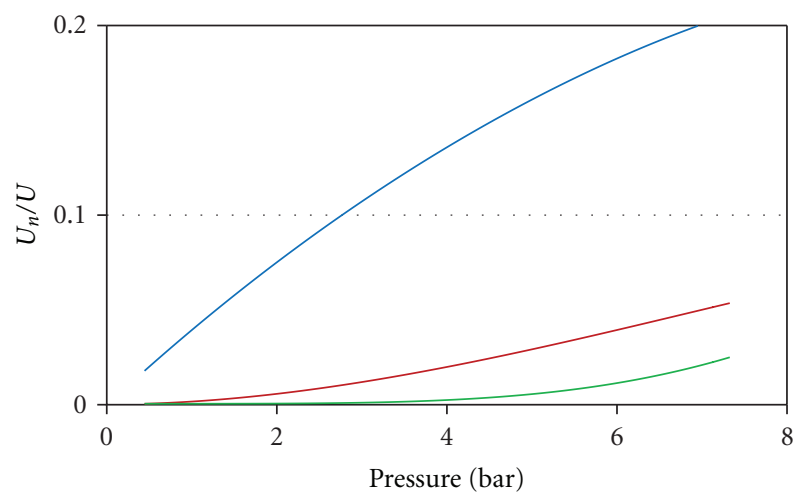

(b)

Figure 5: Shares of the partial potential energies $U_{n}$ in the total potential energy $U$ of the Water vapor. Blue lines: $n=3$; red lines: $n=4$; green lines: residuals; (a) $T=290 \mathrm{~K}$; (b) $T=440 \mathrm{~K}$.

We assume for the product $U D$ an equation:

$$
U D=-\sum\left(E_{n} C_{\mathrm{un}} D_{m}^{n}\right) .
$$

To find the coefficients $K_{\mathrm{un}}=C_{\mathrm{un}} E_{n}$, at $K_{u 1}=0$, we expand the value $U D / D_{m}^{2}$ by $D_{m}$ to the second exponent of $D_{m}$. It gives contributions to potential energy of small cluster fractions with a number of particles in a cluster up to four. A special method for this series expansion was developed to take into account the higher-order residual terms of the series expansion. The maximal density was limited by the criterion: the residual term should be small as compared to the found terms of expansion at this density. For example, the density for Argon gas was limited at the $0.3 \mathrm{~mol} / \mathrm{L}$ level.

The mathematical aspects of this method stay too far from the paper on physical aspects of molecular interactions in real gases and may be described separately. The typical results of the series expansion for the Water vapor potential energy are shown at the Figure 5. The Figure shows the pressure dependences for shares of the trimers' and tetramers' fractions in the total potential energy at low and high temperatures. For comparison the share of the residual terms has been also plotted.

It is seen that, at $T=290 \mathrm{~K}$, the share of tetramers is much larger than the trimers' share and reaches almost $10 \%$. The residuals' share at this temperature is much lower than

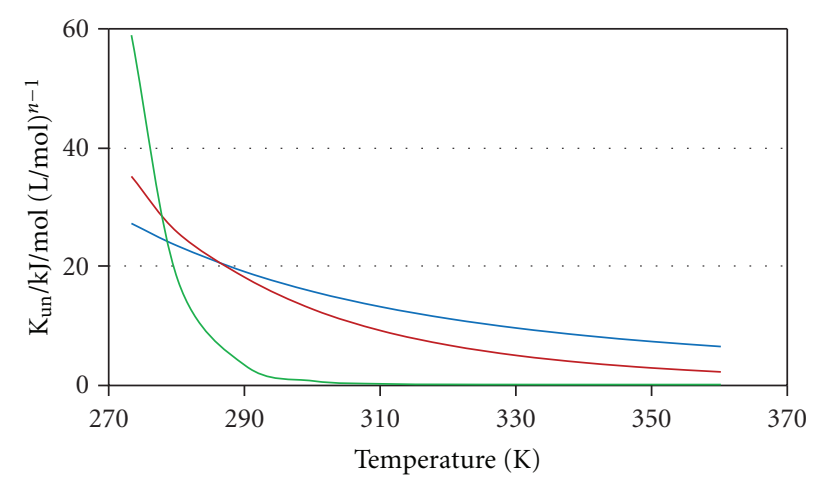

FIGURE 6: The series expansion coefficients for unit volume potential energy of the Water vapor. Blue line: $K_{u 2}(T)$; red line: $K_{u 3}(T) / 10$; green line: $K_{u 4} / 10^{6}$.

the trimers' share. At $T=440 \mathrm{~K}$, the share of trimers is much larger than the tetramers' share and exceeds $20 \%$. The residuals' share at this temperature is twice lower than the tetramers' share but quickly grows when pressure approaches its saturation value. Small values of residuals confirm the correctness of the expansion method in the temperature, range $273.16-440 \mathrm{~K}$ for pressures up to the saturation level. For a larger temperature the upper limit of pressure should be lower than its saturation value.

The resulting series expansion coefficients for the Water vapor are shown at Figure 6.

Figure 6 shows that near the triple point the potential energy of the Water vapor contains a large contribution from the 4-particle cluster fraction. For this computation, the data from the NIST database [7] have been downloaded with a precision of 10 digits. The database [7] contains the thermodynamically averaged experimental data. Therefore, all statistical processing of the experimental data had been performed at the database preparation. The correctness of this method has been confirmed by the correlation of different thermophysical values: partial energies, pressures, densities, activation energies, clusters' bond energies, and bond volumes, Joule-Thomson coefficients in wide ranges of $T$ and $P$.

3.2. Activation Energies for Cluster Fractions. The temperature dependence of the clusters' $\mathrm{PE}$ equilibrium constants $C_{u n}$ shows that they correspond to real physical clusters, being analogues to chemical compounds. Figure 7 confirms the Boltzmann law for the temperature dependences of the expansion coefficients $K_{u n}$ for partial potential energies in rather wide temperature ranges for the Water vapor and Carbon Monoxide gas.

Figure 7 shows that it is possible to find the activation energies for rather wide temperature ranges that are given by tangents of slopes of the $\ln \left(K_{u n}\right)$ versus $1 / T$ dependence. Figure $7(\mathrm{~b})$ gives three values for activation energies $E_{n}$ of the cluster fractions in the Carbon Monoxide gas. It is seen that the $E_{3} / E_{2}$ ratio is around 2.5 that signals about a complex binding of the third molecule to the dimer with an average number of bonds more than one. But the value for the $E_{4} / E_{2}$ 


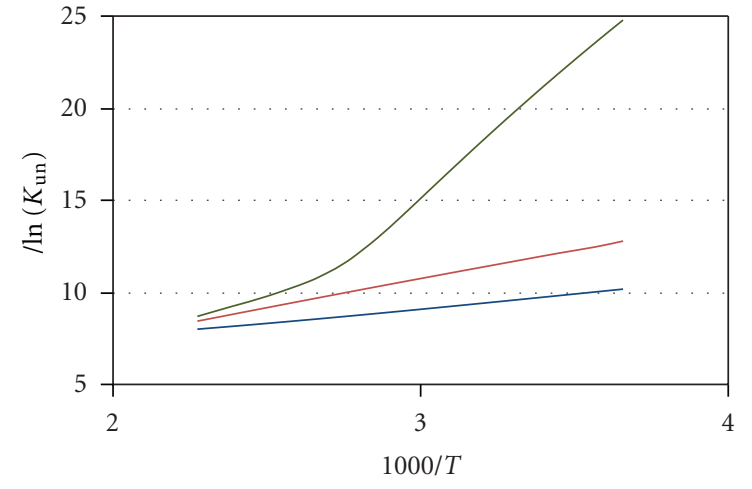

(a)

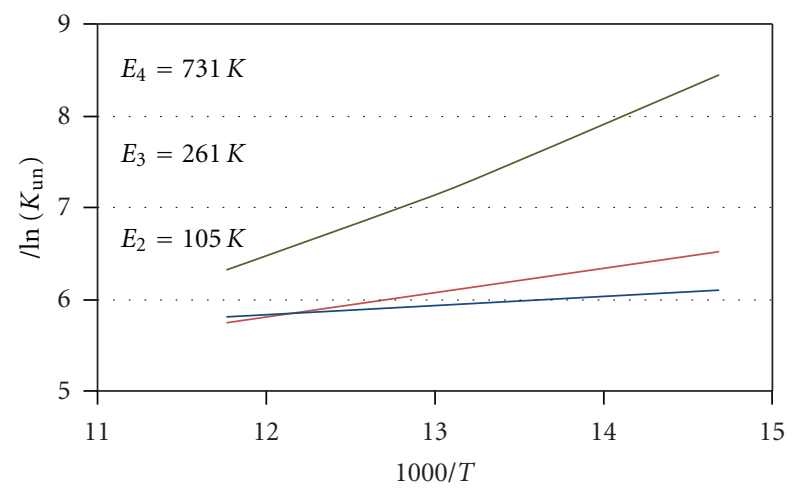

(b)

Figure 7: Linear dependences of the $\ln \left(K_{u n}\right)$ on the reverse temperature 1000/ $T$ for the Water vapor and Carbon Monoxide, corresponding to the Boltzmann law: (a) $\mathrm{H}_{2} \mathrm{O}$ at $T$ between 273.16 and $440 \mathrm{~K}$; (b) $\mathrm{CO}$ at $T$ between 68.16 and $85 \mathrm{~K}$.

ratio near 7 signals about a tightly bonded structure of the tetramers in the $\mathrm{CO}$ gas near the triple point.

A similar picture is in the Water vapor at temperatures lower than $360 \mathrm{~K}$, Figure $7(\mathrm{a})$. The $E_{3} / E_{2}$ ratio is around 2 that signal about an open chain structure of trimers with two Hydrogen bonds, but the $E_{4} / E_{2}$ ratio near 8 signals about a tightly bonded, solid-like, tetramers structure in the $\mathrm{H}_{2} \mathrm{O}$ vapor near the triple point.

The diagram for the Water vapor reflects a very interesting phenomenon: the transformation of tightly bonded tetramers in the open chain tetramers at the temperature range $360-380 \mathrm{~K}$. It is not a phase transition in tetramers: it is a substitution of one predominant isomer type by another, with smaller bond energy, but much larger number of bound states. This substitution is not abrupt but takes almost $20 \mathrm{~K}$ for the temperature zone, where different isomers coexist in a mixed isomer fraction. So the phenomenological approach may give some vision of the microscopic structure of clusters. It could be useful to proceed this research in the mutually coordinated directions: from the molecular dynamics simulations and from the thermophysical properties analysis.

3.3. Equilibrium Constants for Physical Clusters. To find the $\mathrm{PE}$ equilibrium constants $C_{u n}$ from the power expansions of the potential energy, we should first find the clusters' effective bond energies $E_{n}$. We can start from the found values of the activation energies $E_{n}$. In the temperature range, where $E_{n}$ is constant,

$$
C_{u n}(T)=\frac{K_{u n}}{E_{n}}
$$

With found clusters' PE equilibrium constants, we can find the cluster fraction's effective volume of the attraction zone $V_{n}$ from the expression for $C_{u n}(T)$ :

$$
C_{u n}(T)=V_{n} \exp \left(\frac{E_{n}}{T}\right)
$$

The applicability of the Boltzmann law to clusters in the corresponding temperature range may be confirmed by the constant value of the found $V_{n}$.

The cluster fractions' equilibrium constants found from the potential energy, PE equilibrium constants, stay close to the chemical compounds' equilibrium constants in the form of their temperature, dependences. These equilibrium constants correspond to real physical clusters. But, at high temperatures there may be some difference between the spectroscopy and potential energy-based equilibrium constants. The spectroscopy data for compounds' concentrations are not sensitive to the repulsions of the compounds' components, while the potential energy of a cluster may feel these repulsions. The method of accounting for the repulsions in the PE equilibrium constant has to be developed.

3.4. The System of Equations for Pressure and Density in Real Gases. Having found the isothermal row of data $D_{m i}\left(T, P_{i}\right)$, we can reverse this dependence and expand the pressure by $D_{m}$ :

$$
P=R T \sum\left(C_{n} D_{m}^{n}\right)
$$

The right side of (13) looks like a sum of partial pressures for $n$-particle cluster fractions. Therefore, the expansion coefficients $C_{n}(T)$ can be considered as apparent equilibrium constants for partial pressures of $n$-particle cluster fractions, at $C_{1}=1$.

Applying (4) to the expansion (13), we come $[17,18]$ to the series expansion for the total density $D$ by powers of the $D_{m}$ :

$$
D=\sum\left(n C_{n} D_{m}^{n}\right)
$$

This equation again looks like the sum of partial densities of basic particles in the $n$-particle cluster fractions. We came to this series expansion not from microscopic considerations, but on the thermodynamics basis. It confirms our decision to consider $C_{n}(T)$ as the apparent PDT equilibrium constants in spite of their strange temperature dependences, as it will be shown later.

3.5. Chemical Equilibrium for Apparent Cluster Fractions. From (14), we see that the apparent $n$-particle cluster 
fraction's molar density $D_{n}$, found from the PDT relations, is equal to

$$
D_{n}=C_{n} D_{m}^{n}
$$

This result corresponds to the chemical equilibrium theory, but, for apparent cluster densities. The apparent $n$-particle cluster fraction's molar density $D_{n}$ is proportional to the nth power of the monomer fraction density $D_{m}$. It should be stressed again that we did not use the microscopic considerations to come to this expression.

So the knowledge of the $D\left(D_{m}, T\right)$ and $P\left(D_{m}, T\right)$ dependences can be used for computation of the apparent PDT equilibrium constants $C_{n}$ by the joint series expansion of the isothermal $D\left(D_{m}, T\right)$ and $P\left(D_{m}, T\right)$ dependences. In this approach, it is impossible to determine the partial contributions of isomers; therefore, $C_{n}$ reflects a joint contribution of all isomers and mainly of the predominant types of isomers, existing at a given temperature in the $n$-particle cluster fraction.

It should be specially noted that the (13) and (14) are the series expansions of $P$ and $D$ by the monomer fraction density $D_{m}$. In this respect, they differ [23] from the well known virial expansions $[1,9-11,24]$ of $P$ by the total density or $D$ by the total pressure.

At small pressures, the difference between $D$ and $D_{m}$ is small that makes possible to use $D$ instead of $D_{m}$ in the series expansion (13). At these conditions, only the contributions of dimers in the total density and pressure are essential. For this reason, the second PDT equilibrium constant $C_{2}$ correlates with the second virial coefficient $B$, namely, $C_{2}=$ $-B$. It is well known [24] that, at the Boyle temperature $T_{B}$, the second virial coefficient $B$ changes its sign. Therefore, the apparent dimers' PDT equilibrium constant $C_{2}$ and apparent dimers' fraction density become negative at $T>T_{B}$.

\section{Parameters of Molecular Interactions in Pure Real Gases}

The physical clusters can be characterized by their effective parameters: the bond energy $E_{n}$ and the attraction zone volume $V_{n}$. The bond energies for any $n$ may be measured in Joule per mole. After dividing the bond energy values by the universal gas constant $R$, we receive their values in $K$. In this paper, the bond energies are estimated in Kelvin just to compare them with the used values of temperature $T$, because the $E_{n} / T$ ratio is very important for the analysis of equilibrium constants. But, for $V_{n}$, the units of measurement depend on $n$ : for $n=2$, it is the molar volume (L/mol); for $n>2$, the dimension of $V_{n}$ is $(\mathrm{L} / \mathrm{mol})^{n-1}$.

4.1. The Clusters' Effective Bond Energy. In some papers, the maximal depth $U_{\min }$ of the potential well in dimers, shown on the Figure 2(a), is considered as the bond energy, but this value never corresponds to the estimated activation energy for the dimer fraction density. For the reason that the energetic levels in the cluster's bound state are spaced very densely and the gaps between them are much smaller than $k T$, the bond energy $E_{n}$ is a thermodynamic average of localized energy levels. To distinguish it from the chemical bond with clearly defined quantum energetic level, it may be named as the effective bond energy. This value corresponds to the estimated activation energy of the cluster fraction density. The $E_{2} / U_{\min }$ ratio depends on the form of the interaction potential $U(r)$.

4.2. The Clusters' Effective Volume of the Attraction Zone. The effective volume of the dimer's attraction zone $V_{2}$ corresponds to the average number of localized states in the attraction zone $N_{b 2}=V_{2} / V_{q}$. This value for the number of bound states in dimers reflects the smooth dependence of the attraction energy on the interparticle distance in conditions of densely spaced energetic levels. It strongly depends on temperature due to the temperature dependence of $V_{q}$ and differs from the well-known chemical compound's number of states that is the quantum degeneration degree of the compound's ground level and does not depend on temperature.

For larger clusters, the average number of bound states $N_{b n}=V_{n} /\left(V_{q}\right)^{n-1}$, where $V_{n}$ is the effective volume of the cluster's attraction zone with its dimension equal to $3(n-1)$. For all pure gases, there exist the relatively large ranges of temperature, in which both $E_{n}$ and $V_{n}$ are almost constant. These constants can be considered as the fixed parameters of the molecular interactions in pure gases. Besides, for the pair molecular interaction the third parameter, the excluded volume, reflecting the average volume of the repulsion zone, may be found. The question about the excluded volumes for clusters is not so clear and waits for its decision.

4.3. The PDT Equilibrium Constants Determination. To remove the large regular part from the $D\left(D_{m}, T\right)$ and to pay more attention to the clusters contributions in it, we expand by the $D_{m}$ the function $\left(D-D_{p}\right) / D_{m}^{2}$. Here, $D_{p}=P / R T$ is the density of ideal gas at pressure $P$. The coefficients of this expansion are $C_{2}(T), 2 C_{3}(T), 3 C_{4}(T), \ldots$ This type of serial expansion can be named as a joint series expansion of the system of (13) and (14), because, during the expansion, we use both equations of this system. This procedure is repeated for different temperatures, and, thus, we receive the temperature dependences of the $C_{n}(T)$. Figure 8 shows the results of the $C_{n}(T)$ computation for Water vapor, $n=2,3,4$.

The presented on Figure 8 results of the computerized analysis of experimental thermophysical data from the NIST database [7] for the Water vapor show that the apparent equilibrium constant for 4-particle cluster fraction is very large and its temperature dependence is very steep. It confirms the made earlier conclusion about a large value of the effective bond energy $E_{4}$ for tetramers in the Water vapors. But, now, it is impossible to find the activation energy because the apparent PDT equilibrium constants do not correspond to the Boltzmann law.

Figure 9 shows that the apparent PDT equilibrium constant is lower than the PE equilibrium constant on almost a constant value. 


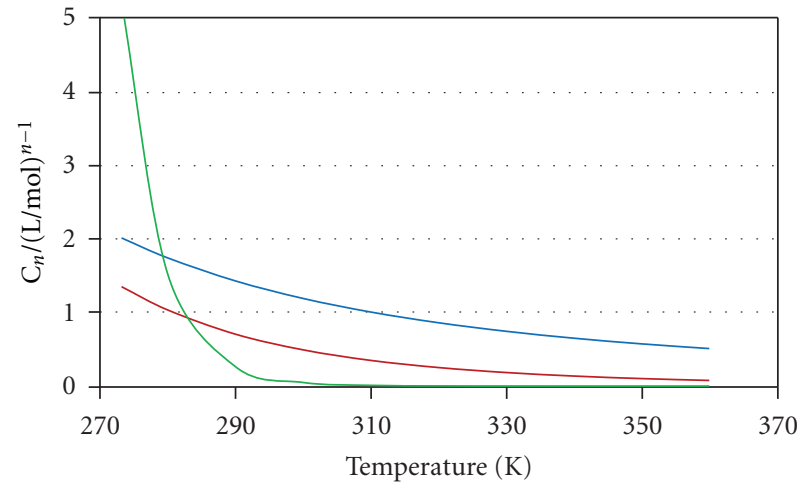

FIgURE 8: The apparent PDT equilibrium constants for the Water vapor at temperatures between the triple and normal boiling points. Blue line: $C_{2}(T)$; red line: $C_{3}(T) / 10$; green line: $C_{4}(T) / 10^{5}$.

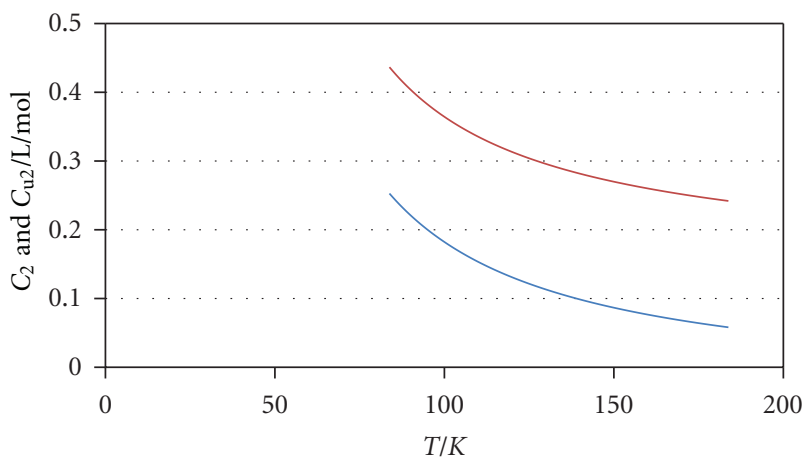

(a)

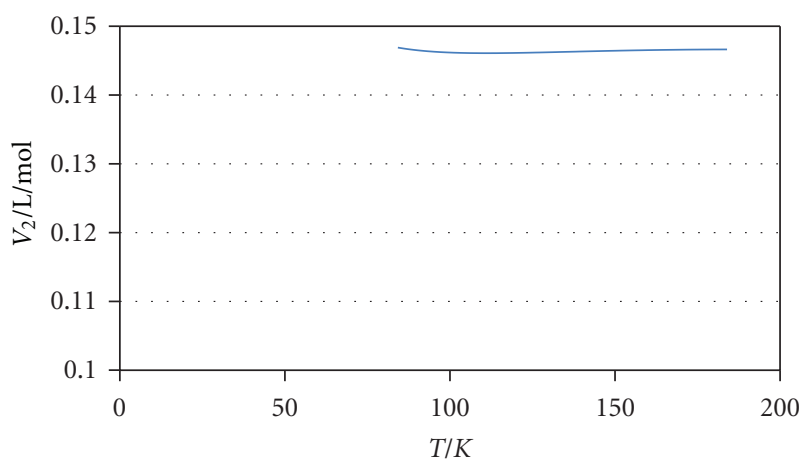

(b)

FIGURE 9: Results of computation of the equilibrium constants and the effective volume of the attraction zone for dimers in Argon. (a) Blue line: the apparent PDT equilibrium constant; red line: the PE equilibrium constant. (b) The effective volume of the attraction zone.

To come to the effective bond energies for different cluster fractions, we should use both the apparent PDT equilibrium constants and the series expansion coefficients for potential energy.

4.4. A New Method for Estimation of the Clusters' Effective Bond Energies. The comparison of the found earlier PE

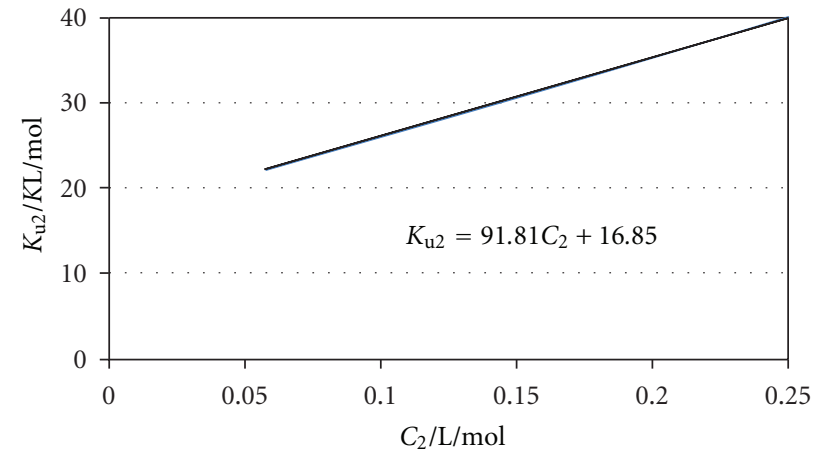

(a)

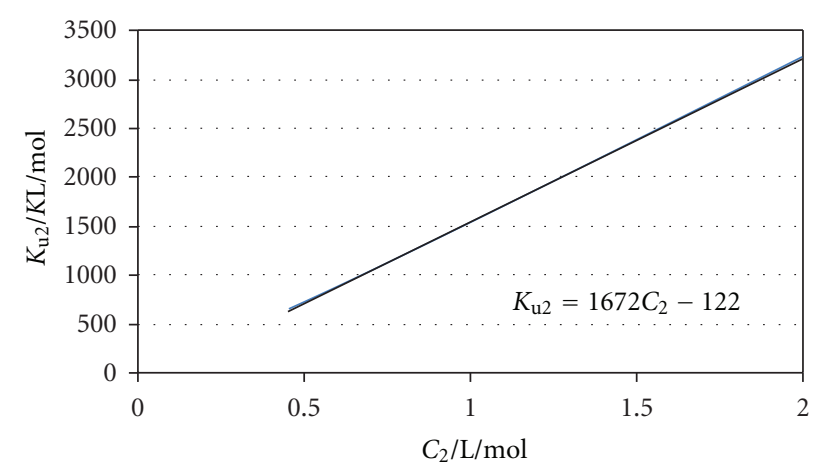

(b)

Figure 10: The correlations between the second-order series expansion coefficients of the potential energy $U D$ for a unit volume and the difference between the real and ideal densities $\left(D-D_{p}\right)$ : (a) for the gaseous Argon in the temperature range between the triple point $T_{t r}$ and $T_{t r}+100 \mathrm{~K}$; (b) for the Water vapor in the temperature range between the triple point and the normal boiling point.

equilibrium constants $C_{u n}$ with the PDT equilibrium constants shows that $C_{n}$ contains in addition to the equilibrium constants $C_{u n}$ corresponding to real physical clusters the virtual part $C_{v n}$ :

$$
C_{n}=V_{n} \exp \left(\frac{E_{n}}{T}\right)+C_{v n} .
$$

The analysis of data for many gases shows that the $C_{v n}$ changes very slightly if compared to the changes of the $C_{n}$ or $C_{u n}$ with temperature. In some temperature ranges, it is almost constant. Therefore, the diagram of the $K_{u n}=C_{u n} E_{n}$ versus $C_{n}$ is close to linear with a tangent of slope equal to $E_{n}$.

To find from the found $K_{u n}(T)$ dependences the effective bond energies $E_{n}$ and the PE equilibrium constants, we plot the $K_{u n}$ versus $C_{n}$. The resulting curve can be named as the correlation curve between the expansion coefficients $K_{u n}$ and $C_{n}$. Figure 10 presents an example of such a plot for dimers in Argon and Water vapors.

Figure 10 shows that the correlation curves for dimers in the Argon and Water vapors in the temperature range between the triple point and the triple point $+100 \mathrm{~K}$ are close to linear. Their tangents of slope give us the averaged for the said temperature ranges, the dimers' effective bond energies $E_{2}$. For these diagrams potential energy was divided by the 
gas constant $R$; therefore, dimension of the $K_{u 2}$ is $(\mathrm{K} \mathrm{L} / \mathrm{mol})$ and the average tangent of slope gives the effective bond energy in $K$. From Figure 10, it is seen that the effective bond energy for dimers in the Argon gas is equal to $91.81 \mathrm{~K}$ and, in the Water vapor, it is equal to $1672 \mathrm{~K}$. This procedure can be repeated for other $n$; thus, we come to the cluster fractions' effective bond energies. For the Water vapor, the results are shown in the Table 1 . The difference of the $E_{2}$ values in the Table 1 and Figure 10(b) results from small nonlinearities of the used dependences.

4.5. Estimation of the Bond Volumes. For the Argon gas, the found values of $V_{2}$ are very close to a constant value equal to $0.146 \mathrm{~L} / \mathrm{mol}$. An additional confirmation gives the activation energy found from the $\ln \left(C_{u 2}\right)$ plot versus $1 / T$ that is close to the found value for $E_{2}=91.81 \mathrm{~K}$. The effective bond energies determination errors result from the nonlinearities of the correlation curves shown on the Figure 10. For dimers in Argon and Water vapor, they do not exceed $2 \%$ in the temperature range under investigation. So we come to the same results for effective bond energies as from the estimations of the activation energy and from the correlation between the PDT and PE expansion coefficients.

For the Water vapor, the found values for the effective volumes $V_{n}$ of the attraction zones and effective bond energies $E_{n}$ in clusters are shown in the Table 1.

\section{The Results of the Molecular Interaction Parameters Computation for Some Gases}

5.1. The Effective Bond Parameters for the Water Vapor. In addition to results for the Water vapor, shown on the Figure 7(a), we present here the values of the effective bond energies and effective volumes of the attraction zones for the temperature range between the triple point and the normal boiling point, see Table 1. The normal boiling point for Water stays near the center of the temperature range, where the exchange of the predominant types of tetramers takes place. So the table gives parameters for the low temperature range, where tetramers have rigid solid-like structure with a large value of the bond energy and with a very small value for the effective volume of the attraction zone.

The shown $E_{n} / E_{2}$ ratios permit to estimate the type of the predominant isomers. The trimers' ratio being near 2 tells about the open chain trimers' structure with two Hydrogen bonds, but the tetramers' ratio near 8 signals about the closed structure with eight Hydrogen bonds. The number of Hydrogen bonds may be smaller, if every bond becomes stronger in the tightly bound cluster. But the conclusion about the solid-like crystalline structure of tetramers in the Water vapor near its triple point is correct at any case. An additional confirmation of this idea is provided by the $V_{n} / V_{2}^{n-1}$ ratio. In trimers, it is around 3 and tells about the possible number of diverse open chain configurations with a large freedom of molecular movements. But, in tetramers, it is very small that signals about the rigid crystalline structure of clusters in this range of temperatures.

These results have been achieved after a large work. But the conclusion about an essential role of tetramers in the
TABLE 1: The effective bond parameters for the Water vapor clusters at $T$ between the triple and normal boiling points.

\begin{tabular}{lccc}
\hline Parameters for $n$ & 2 & 3 & 4 \\
\hline Bond energy $E_{n}, K$ & 1690 & 3370 & 13500 \\
$E_{n} / E_{2}$ ratio & & 1.99 & 7.99 \\
Bond volume $V_{n}(\mathrm{~L} / \mathrm{mol})^{\mathrm{n}-1}$ & $3.910^{-3}$ & $4.710^{-5}$ & $1.410^{-16}$ \\
$V_{n} / V_{2}^{\mathrm{n}-1}$ ratio & & 3.1 & $2.410^{-9}$ \\
\hline
\end{tabular}

Water vapor near the triple point may be seen directly from the density dependence of the Joule-Thomson coefficient. This coefficient may give very useful information about molecular interactions in gases.

5.2. The Joule-Thomson Coefficient for the Water Vapor. The shown on Figures 6 and 8 large values of equilibrium constants for tetramers in the Water vapor are reflected in the Joule-Thomson coefficient $\mu$ density dependence. According to Jame Wisniak [25], "the Joule-Thomson coefficient may be calculated from a knowledge of the PVT relationship of the fluid (equation of state) and the specific heat at constant pressure":

$$
\mu=\frac{1}{C_{p}}\left(\left.T\left(\frac{\partial V}{\partial T}\right)\right|_{P}-V\right) .
$$

It is useful to remove large regular part from $V$ by introduction of the interaction volume $V_{\text {int }}=R T / P-V$. After that, (17) may be rewritten as

$$
\mu C_{p}=V_{\text {int }}-\left.T\left(\frac{\partial V_{\text {int }}}{\partial T}\right)\right|_{P}
$$

The interaction volume is proportional to the used in our series expansion value $\left(D-D_{p}\right)$ :

$$
V_{\text {int }}=\frac{D-D_{p}}{D D_{p}}
$$

In the Water vapor, the difference between $D, D_{p}$ and $D_{m}$ is very small. Therefore, it is possible to change the denominator of (19) on the $D_{m}^{2}$. Then, the $V_{\text {int }}$ becomes just the value $\left(D-D_{p}\right) / D_{m}^{2}$ that was used in the series expansion to find the PDT equilibrium constants. According to the system (13) and (14),

$$
\frac{D-D_{p}}{D_{m}^{2}}=C_{2}+2 C_{3} D_{m}+3 C_{4} D_{m}^{2}+\cdots .
$$

Therefore, we can transform (18) in the series expansion for the $\mu C_{p}$ :

$$
\begin{aligned}
\mu C_{p}= & C_{2}-\frac{T d C_{2}}{d T}+2\left(C_{3}-\frac{T d C_{3}}{d T}\right) D_{m} \\
& +3\left(C_{4}-\frac{T d C_{4}}{d T}\right) D_{m}^{2} .
\end{aligned}
$$




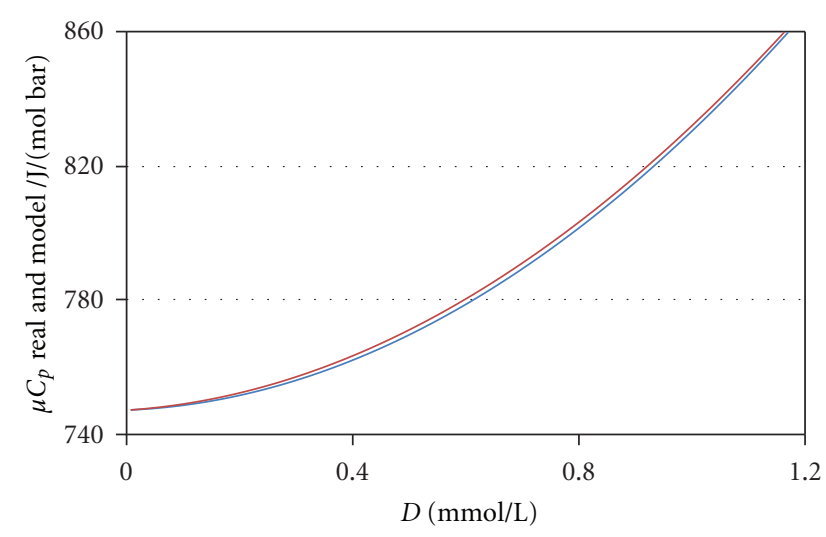

(a)

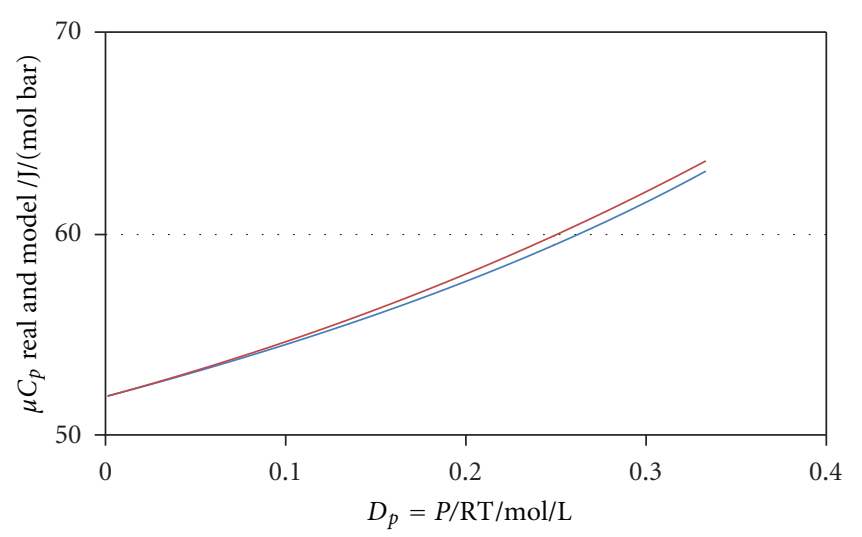

(b)

FIgURE 11: The product of the Joule-Thomson coefficient by specific heat at constant pressure versus molar density: (a) in the Water vapor at temperature $300 \mathrm{~K}$, (b) in the Argon gas at temperature $100 \mathrm{~K}$. Blue lines: experimental values; red lines: modeled values by (22) and (24).

We know that $C_{2}(T)$ differs from the $C_{u 2}(T)=V_{2} \exp \left(E_{2} / T\right)$ on a constant value $C_{v 2}$ that gives zero at differentiation. So we finally have the expression for the $\mu C_{p}$ :

$$
\mu C_{p}=C_{2}+\frac{K_{u 2}}{R T}+2\left(C_{3}+\frac{K_{u 3}}{R T}\right) D_{m}+3\left(C_{4}+\frac{K_{u 4}}{R T}\right) D_{m}^{2} .
$$

The expression (22) predicts a large quadratic density dependence of the $\mu C_{p}$ product in the Water vapor. This prediction is confirmed by Figure 11(a). Equation (22) is valid for gases with large $E_{n} / T$ ratios, like Water or Methanol vapors. But for van der Waals gases, like Argon, the expression for $\mu C_{p}$ should take into account the difference between monomer fraction density and total density of the gas.

The quadratic character of the $\mu C_{p}$ dependence on density, shown on Figure 11(a), tells about a large share of 4-particle clusters in the Water vapor at room temperatures.
TABLE 2: The values of apparent equilibrium constants $C_{n}$ and series expansion coefficients $K_{u n}$ for potential energy of the Argon gas at $T=100 \mathrm{~K}$.

\begin{tabular}{ccccccc}
\hline$n$ & $C_{n}$ & Units & Values & $K_{u n}$ & Units & Values \\
\hline 2 & $C_{2}$ & $\mathrm{~L} / \mathrm{mol}$ & 0.182778 & $K_{u 2}$ & $\mathrm{JL} / \mathrm{mol}^{2}$ & 279.3154 \\
3 & $C_{3}$ & $(\mathrm{~L} / \mathrm{mol})^{2}$ & 0.066657 & $K_{u 3}$ & $\mathrm{JL}^{2} / \mathrm{mol}^{3}$ & 215.5387 \\
4 & $C_{4}$ & $(\mathrm{~L} / \mathrm{mol})^{3}$ & 0.036931 & $K_{u 4}$ & $\mathrm{JL}^{3} / \mathrm{mol}^{4}$ & 185.4765 \\
\hline
\end{tabular}

5.3. The Joule-Thomson Coefficient for Van Der Waals Gases. The expansion of the $V_{\text {int }}$, given by (19), with account of (13) and (14), up to the second exponent of the $D_{m}$ results in

$$
V_{\text {int }}=C_{2}+\left(2 C_{3}-3 C_{2}^{2}\right) D_{m}+\left(3 C_{4}-10 C_{3} C_{2}+5 C_{2}^{3}\right) D_{m}^{2} .
$$

The differentiation of equilibrium constants by $T$ gives $T d C_{n} / d T=-K_{u n} / R T$. The differentiation of $D_{m}$ gives $\left.T\left(\partial D_{m} / \partial T\right)\right|_{P}=-\left(K_{u 2} / R T+D_{p} / D\right) D_{p} / T$. In the result, we have

$$
\begin{aligned}
\mu C_{p}= & C_{2}+\frac{K_{u 2}}{R T}+2\left(C_{3}+\frac{K_{u 3}}{R T}-3 C_{2}^{2}-6 C_{2} \frac{K u 2}{R T}\right) D_{m} \\
+ & \left(3\left(C_{4}+\frac{K u 4}{R T}\right)-10 C_{3} C_{2}+5 C_{2}^{3}\right. \\
& \left.\quad-10\left(C_{3} \frac{K_{u 2}}{R T}+C_{2} \frac{K_{u 3}}{R T}\right)\right) D_{m}^{2}+15 C_{2}^{2} \frac{K_{u 2}}{R T} D_{m}^{2} \\
+ & \left(\left(2 C_{3}-3 C_{2}^{2}\right)+2\left(3 C_{4}-10 C_{3} C_{2}+5 C_{2}^{3}\right) D_{m}\right) \\
& \times\left(\frac{K_{u 2}}{R T}+\frac{D_{p}}{D}\right) \frac{D_{p}}{T} .
\end{aligned}
$$

To apply this equation to some real gas, for example, Argon, we should know the values of apparent equilibrium constants and coefficients of the potential energy expansion at some fixed temperatures. For Argon, we have the values for expansion coefficients at $T=100 \mathrm{~K}$, shown in Table 2 .

The result of these values substitution into (19) is shown on Figure 11(b). The deviations of the predicted values from the experimental values on Figure 11 can be explained by the assumptions made in the derivation of the (22) and (24).

5.4. Molecular Interaction Parameters for Gaseous Alkanes. Applying the described method of equilibrium constants and their parameters determination to the row of Alkanes, we met some deviations of their characteristics from those described in the previous section. First of all, the correlation curve of $K_{u 2}$ and $C_{2}$ does not look like the straight line. That is clearly seen on Figure 12.

Figure 12 shows that the forms of the correlation curve $K_{u 2}\left(C_{2}\right)$ for different representatives of the Alkanes are similar, only the maximal values of the PDT equilibrium constants grow with the number of carbon atoms in the molecule. It is rather understandable.

But if we compare these correlation curves with curves shown on Figure 10 for Argon and Water, we can notice that the sign of the curvature is opposite. In Alkanes, the tangent 


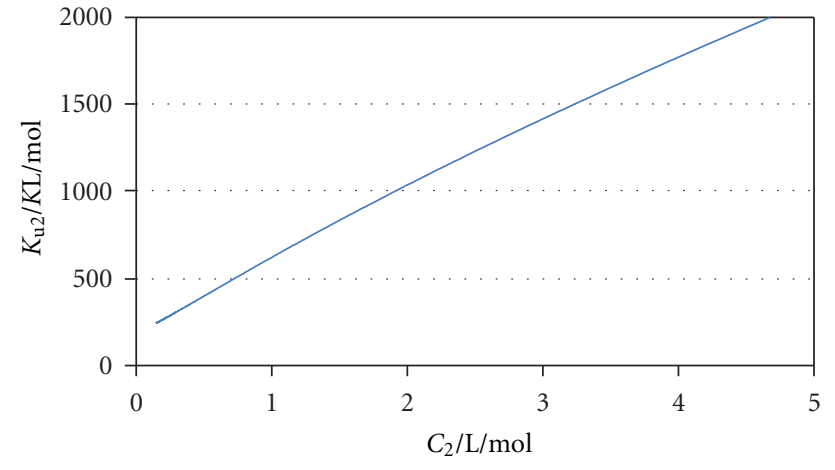

(a)

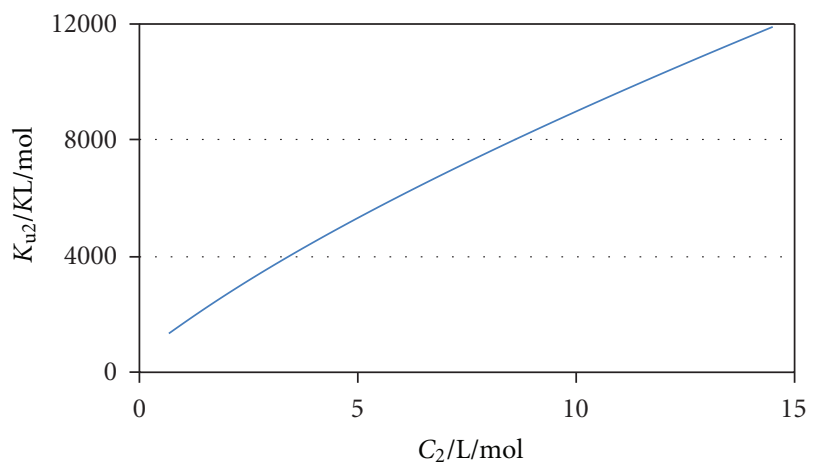

(b)

FIgURE 12: Typical correlation curves $K_{u 2}\left(C_{2}\right)$ for Alkanes: (a) the correlation curve for Butane; (b) the correlation curve for Decane.

of the curve's slope at low temperatures becomes lower than at higher temperatures. In many other gases, including Argon and Water, there is a growth of the tangent to lower temperatures. The growing tangent at low temperatures is understandable. It tells about the possible growth of the effective bond energy at low temperatures. But the apparent diminishing of the effective bond energy in Alkanes at low temperatures may signal about some unknown detail of molecular interactions in these gases.

Therefore, the effective interaction parameters have been estimated only for high temperatures, where the correlation curves become straighter. The results are shown on the Figure 13.

Figure 13 shows that, in Alkanes, there is almost linear dependence of the effective bond energy on the number of carbon atoms in the molecule. But this line doubles: for even numbers of $\mathrm{C}$ atoms, the bond energies are slightly higher than for odd numbers. The zig-zag dependence of the Alkanes' properties on the number of $\mathrm{C}$ atoms $n$ in their molecules is well known. The melting points for the row of Alkanes from Ethane to Decane [1] plotted versus $n$ show also the zig-zag dependence, Figure 14. This paper has a goal to develop new methods of the experimental thermophysical properties analysis. The author hopes that the parallel between the zig-zag dependences for the effective pair bond energy and melting point in Alkanes may stimulate new research works in this direction.

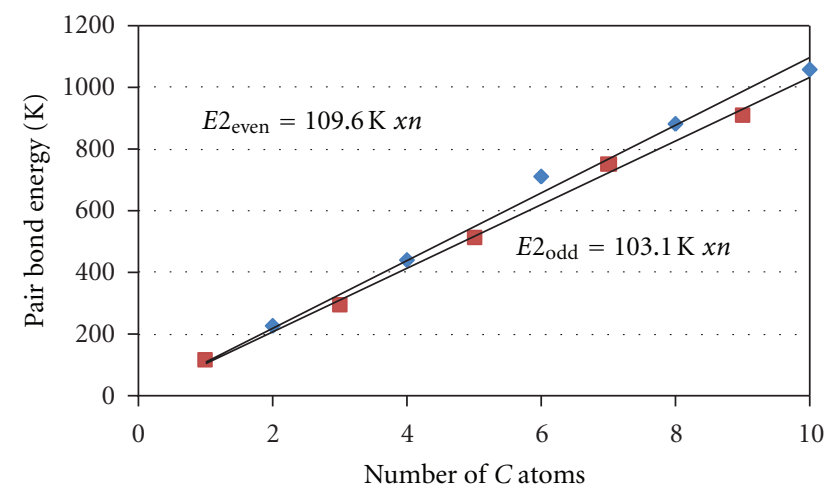

(a)

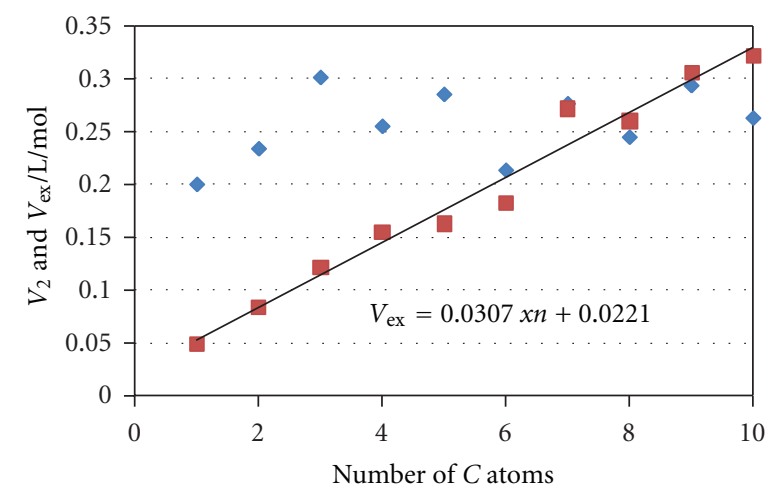

(b)

FIGURE 13: Effective pair bond parameters versus the number $n$ of $\mathrm{C}$ atoms in Alkanes molecules: (a) effective pair bond energy $E_{2}(n)$ : blue rhombs-even numbers; red squares-odd numbers; (b) effective volume for the attraction zone $V_{2}(n)$ : blue rhombs; Excluded volume $V_{\text {ex }}(n)$ : red squares.

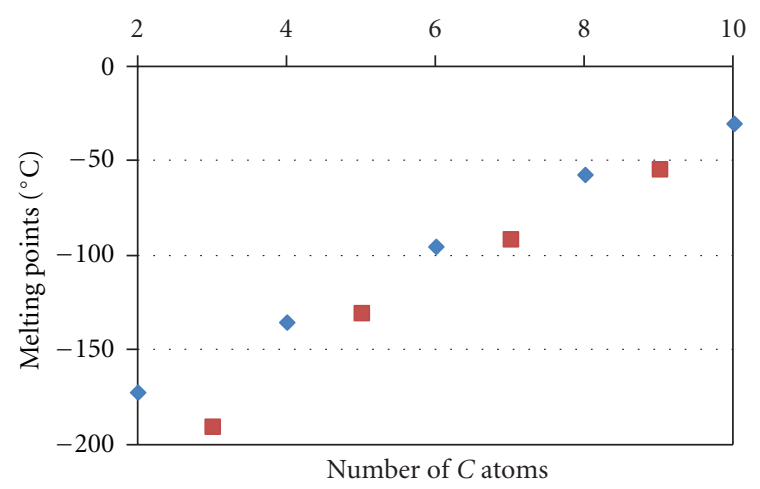

FIGURE 14: Melting points of Alkanes versus the number $n$ of $\mathrm{C}$ atoms in molecules.

The values of the attraction zone volume $V_{2}$, shown at Figure 13(b), do not exhibit a general dependence on the number of $\mathrm{C}$ atoms but have a large dispersion.

The excluded volume grows linearly with growing own volume of molecules that is quite natural.

These facts permit to make conclusions about the predominant dimer configuration in Alkanes. Linear growth of the bond energy with the number of $\mathrm{C}$ atoms tells about the 
parallel position of molecules in the dimer, when all $\mathrm{C}$ atoms participate equally in the interaction. The value of the $V_{2}$ being almost constant also tells in favor of this configuration: the interacting molecules move along their axes not more than on the $\mathrm{C}-\mathrm{C}$ distance, independently on the number of $\mathrm{C}$ atoms.

\section{Conclusions}

(i) It was shown that, for real gases, there is an essential difference between the apparent clusters' equilibrium constants responsible for pressure and potential energy.

(ii) The dimer equilibrium constant for potential energy of a real gas looks like the equilibrium constant for molecular compounds and its temperature dependence obeys to the Boltzmann law. Therefore, it corresponds to real physical dimers.

(iii) The dimer equilibrium constant for pressure contains an additional negative term that may be attributed to virtual dimers. Due to this term, it crosses the zero level and becomes negative at temperature over the Boyle point.

(iv) The introduction of the monomer fraction density in a family of thermodynamics variables expands power of the computer-assisted analysis of thermophysical properties of pure gases.

(v) The developed method of the computer-aided analysis of the precise thermophysical values from the NIST databases shows the existence in the Water vapor of tetramers with large bond energy at temperatures lower than the boiling point.

(vi) The investigation of the molecular interactions in Alkanes resulted in the linear dependence of the effective bond energy on the number of $\mathrm{C}$ atoms in molecule and led to conclusions about parallel position of molecules in the Alkanes dimers.

\section{References}

[1] L. Pauling, General Chemistry, W.H. Freeman and Company, San-Francisco, Calif, USA, 1970.

[2] O. Sackur, "Die universelle Bedeutung des sog. elementaren Wirkungsquantums," Annalen der Physik, vol. 40, p. 67, 1913.

[3] H. Tetrode, "Die chemische Konstante der Gase und das elementare Wirkungsquantum," Annalen der Physik, vol. 343, no. 7, pp. 434-442, 1912.

[4] C. T. Ewing, "Molecular association in sodium, potassium, and cesium vapors at high temperatures," Journal of Physical Chemistry, vol. 71, no. 3, pp. 473-477, 1967.

[5] N. B. Vargaftik, Yu. K. Vinogradov, and V. S. Yargin, Handbook of Physical Properties of Liquids and Gases, Begell House, 3rd edition, 1996.

[6] Q. Yu and H. Gao, "A simple determination of the $\mathrm{NO}_{2}$ dimerization equilibrium constant," Journal of Chemical Education, vol. 74, no. 2, pp. 233-234, 1997.

[7] NIST Database on Thermophysical Properties of Fluids, http://webbook.nist.gov/chemistry/fluid.
[8] NIST Database of the Thermophysical Properties of Gases Used in the Semiconductor Industry, http://properties.nist .gov/fluidsci/semiprop/.

[9] L. D. Landau and E. M. Lifshitz, Statistical Physics, Pergamon Press, London, UK, 1980.

[10] R. Feynman, Statistical Mechanics; A Set of Lectures, W.A. Benjamin, Mass, USA, 1972.

[11] J. E. Mayer and G. M. Mayer, Statistical Mechanics, John Wiley and Sons, New York, NY, USA, 1977.

[12] I. J. Ford, "Statistical mechanics of nucleation: a review," Journal of Mechanical Engineering Science C, vol. 218, no. 8, pp. 883-899, 2004.

[13] P. Schaaf, B. Senger, and H. Reiss, "Defining physical clusters in nucleation theory from the N-particle distribution function," Journal of Physical Chemistry B, vol. 101, no. 43, pp. 87408747, 1997.

[14] I. Kusaka and D. W. Oxtoby, "Identifying physical clusters in vapor phase nucleation," Journal of Chemical Physics, vol. 110, no. 11, pp. 5249-5261, 1999.

[15] D. I. Zhukhovitskii, "Structural transition in hot small clusters," Journal of Chemical Physics, vol. 110, no. 16, pp. 7770-7778, 1999.

[16] IUPAC, Compendium of Chemical Terminology, The Gold Book, Blackwell Scientific Publications, Oxford, UK, 2nd edition, 1997.

[17] B. Sedunov, "Monomolecular fraction in real gases," in Proceedings of the Joint European Thermodynamics Conference (JETC IX '07), pp. 176-180, 2007.

[18] B. Sedunov, "Monomer fraction in real gases," International Journal of Thermodynamics, vol. 11, no. 1, pp. 1-9, 2008.

[19] B. Sedunov, "New approaches to teaching the thermal physics of fluids," in Proceedings of the 21st IUPAC International Conference on Chemical Thermodynamics (ICCT '10), p. 339, Tsukuba, Japan, 2010.

[20] Ch. Kittel, Thermal Physics, John Wiley and Sons, New York, NY, USA, 1970.

[21] G. N. Lewis and M. Randall, Thermodynamics, McGraw-Hill, New York, NY, USA, 2nd edition, 1961.

[22] L. Belloni, "A Note on fermi's route to fermi-dirac statistics," Scientia, vol. 113, pp. 422-430, 1978.

[23] B. Sedunov, "Equilibrium constants for clusters in real gases," in Proceedings of the 23rd ESAT, p. 66, Cannes, France, 2008.

[24] R.C. Reid, J. M. Prausnitz, and T. K. Sherwood, The Properties of Liquids and Gases, McGraw-Hill, New York, NY, USA, 1977.

[25] J. Wisniak, "The Joule-Thomson coefficient for pure gases and their mixtures," Journal of Chemical Education, vol. 4, pp. 5157, 1999. 

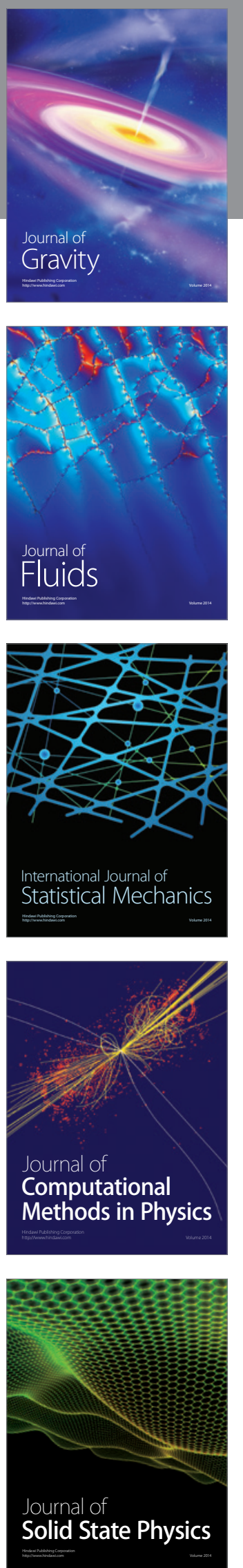

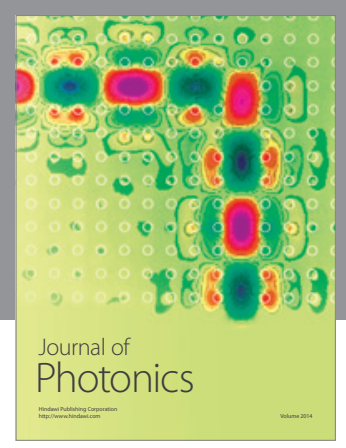

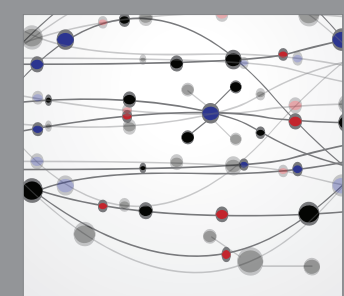

The Scientific World Journal
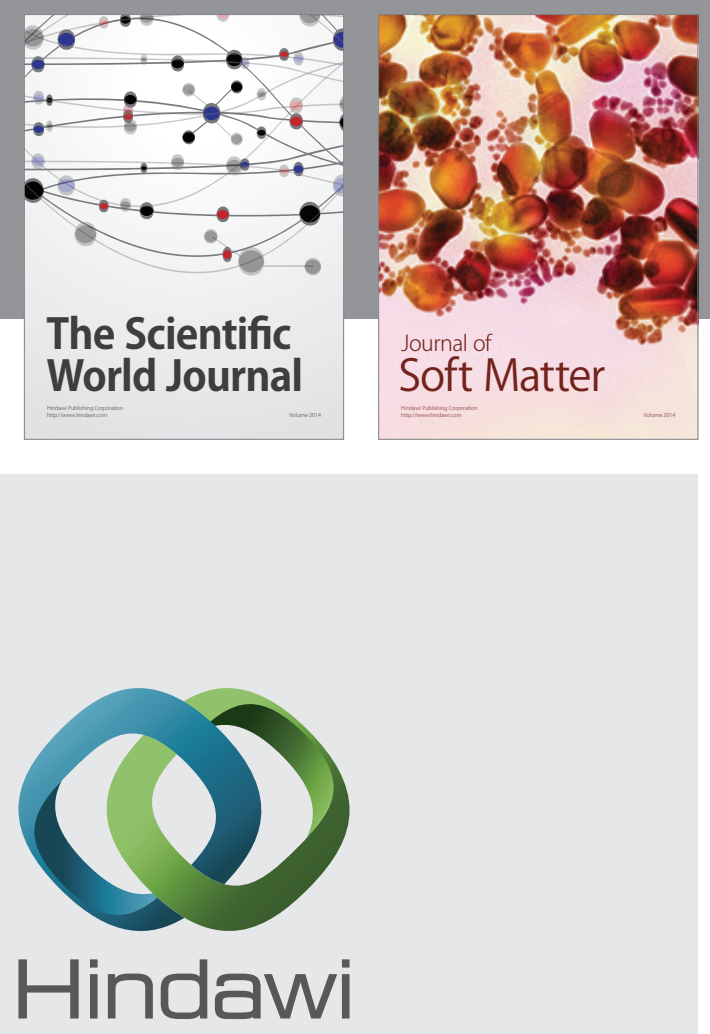

Submit your manuscripts at

http://www.hindawi.com
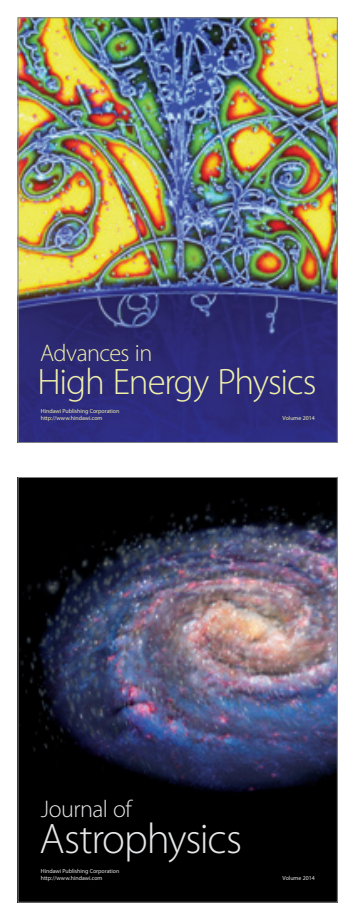
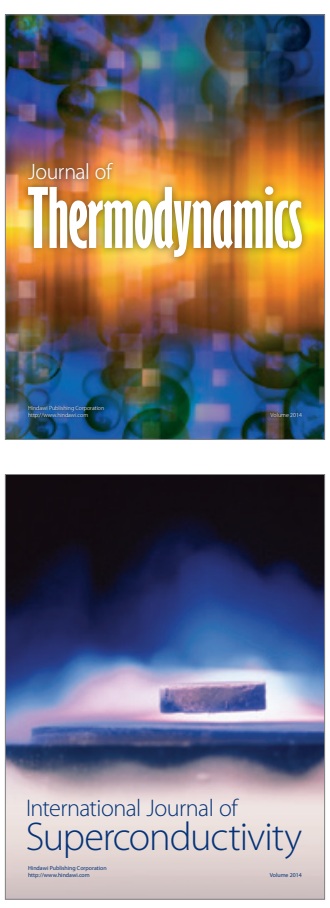
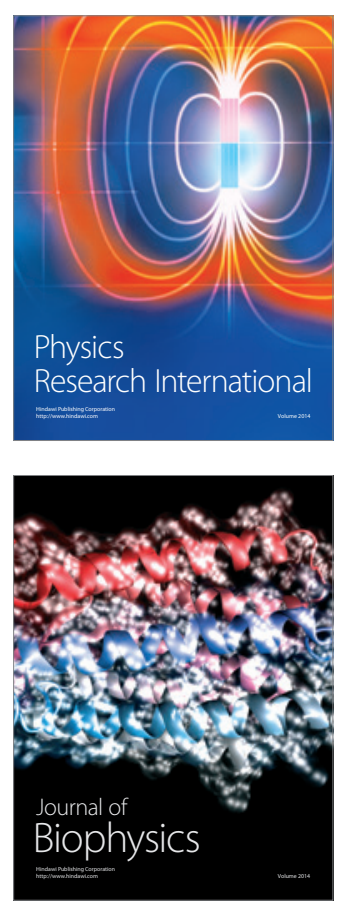
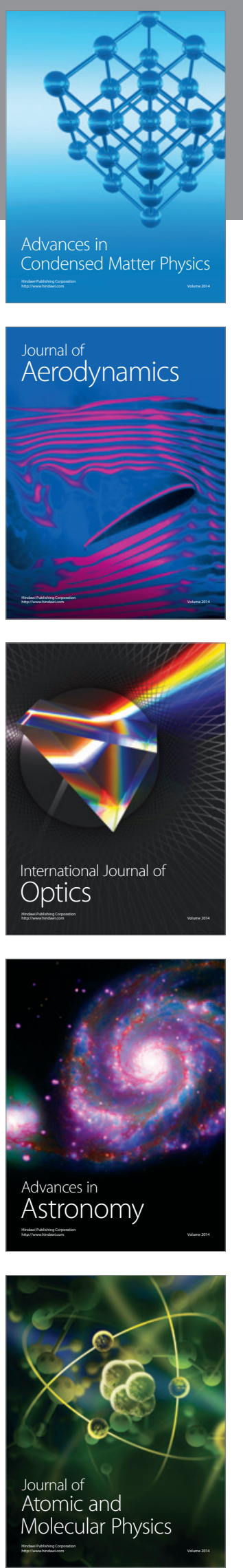